\title{
Magnetic Contour Maps at the Core-Mantle Boundary
}

\author{
Edward R. Benton, ${ }^{*}$ Lorant A. Muth, ${ }^{* *}$ and Michael Stix*** \\ *Department of Astro-Geophysics, University of Colorado, \\ Boulder, Colorado 80309, U.S.A. \\ **Department of Physics and Astrophysics, University of Colorado, \\ Boulder, Colorado 80309, U.S.A. \\ ***Kiepenheuer-Institut für Sonnenphysik, Schöneckstrasse 6, \\ D-7800 Freiburg im Breisgau, West Germany
}

(Received November 9, 1978; Revised May 4, 1979)

\begin{abstract}
Contour maps of the northward, eastward, and downward components of the main geomagnetic field and of the field intensity at the surface of earth's liquid core are presented and compared with similar maps at earth's surface. The mantle is assumed to be an insulator for purposes of the extrapolation. Maximum values of the individual field components are found to be amplified by factors of order 10-20 and the maximum field intensity by 12 over the value at earth's surface and the increase in small scale structure is evident. By comparing maps of places where $B_{r}=0$ at different truncation levels, we find that even for a recent magnetic model which best fits the data quite well, contours of the field extrapolated to the core are quite sensitive to truncation level. This suggests a need to develop nonlinear data-fitting procedures if one's interest is in precise location of field contours rather than in estimating field values.
\end{abstract}

\section{Introduction}

Observations of the main geomagnetic field obtained at or near earth's surface (assumed here to be a sphere of radius $r=a=6.3712 \cdot 10^{6} \mathrm{~m}$ ) are normally represented by a truncated spherical harmonic expansion for the magnetic scalar potential $V$ :

$V(r, \theta, \phi, t ; N)=a \sum_{n=1}^{N} \sum_{m=1}^{n}\left(\frac{a}{r}\right)^{n+1}\left[g_{n}^{m}(t) \cos m \phi+h_{n}^{m}(t) \sin m \phi\right] \cdot P_{n}^{m}(\cos \theta)$.

Here $\theta, \phi, t$ are co-latitude, longitude, time; $P_{n}^{m}$ is the Schmidt quasi-normalized associated Legendre function, $N$ gives the truncation level, and the Gauss coefficients, $g_{n}^{m}, h_{n}^{m}$, have units of tesla. The complex three-dimensional structure of the field is then presented graphically in terms of isomagnetic contour maps, with components of the magnetic field, $\boldsymbol{B}$, derived from $\boldsymbol{B}=-\nabla V$. Typical of such maps are Figs. 1, 3, 5, and 9 of the World Magnetic Survey (Zmuda 1971, 
p. 190, 192, 194, 198) which are replotted here as Figs. 1a-1d. These figures portray the International Geomagnetic Reference Field (IGRF) for epoch 1965.0 as seen at the surface of the earth.

In this paper, we present and discuss the same isomagnetic contour maps but evaluated instead, by extrapolation of $V$, at earth's core-mantle boundary, taken to be a sphere of radius $r=b=3.485 \cdot 10^{6} \mathrm{~m}$ (ENGDAHL and JoHnson, 1974). Our motivation for doing so stems from a number of considerations. Firstly, dynamo theory is rapidly advancing to the point where realistic models of the geomagnetic dynamo process are becoming feasible (e.g., Moffatt, 1976; SowARD and Roberts, 1976; Busse, 1978, for recent reviews). For this purpose, an acceptable model of hydromagnetism in the core must produce a magnetic field which joins smoothly at the surface of the core onto the observed nearly current free magnetic field in the mantle. Thus, $\boldsymbol{B}$ at the core-mantle interface constitutes one important boundary condition for the geodynamo problem so modelers need to be aware of the magnetic field structure at that location. Secondly, the strength of the toroidal field in the core continues to be an important quantity in dynamo models. Busse (1975), for example, has developed a hydromagnetic geodynamo model which rests heavily on the assumption that the Lorentz force is weak compared with the Coriolis force. This model predicts that the toroidal field is comparable with the poloidal field, rather than greatly exceeding it as in other models. From the extrapolated potential field at the core surface, it is difficult to say anything directly about the toroidal field in the core, but we can at least make a new evaluation of the poloidal fields at $r=b$. When a recent high quality field model of high order (BARRAClOUgh et al., 1975) is utilized, we obtain a maximum field strength significantly larger than the value usually quoted in the literature. A third motivation for this work arises from the independent recognition that substantial information about the horizontal core fluid motion adjacent to the coremantle boundary can, in principle, be extracted from contour maps of the type exhibited here (this rests on earlier work by Roberts and ScotT, 1965; BACKUS, 1968; BoOKer, 1969; plus extensions to it currently in progress, e.g., Benton, 1979). Finally, it must be recognized that extrapolation of the magnetic scalar potential (and its spatial derivatives) from earth's surface towards the sources of the field in the core constitutes an ill-posed problem in the mathematical sense. This is usually expressed in terms of the uncertainty in Gauss coefficients of the field model and the amplification of that uncertainty upon extrapolation to the core (Roberts and Scott, 1964; Roberts and Soward, 1972; Lowes, 1974). Moreover, even if the Gauss coefficients were known perfectly, the speed of convergence of the truncated series for $V$ is still markedly reduced at the core surface compared to the rate of convergence evident at earth's 
surface. Both of these effects can be lumped together conceptually in terms of a set of effective Gauss coefficients $(a / b)^{n+1} g_{n}^{m},(a / b)^{n+1} h_{n}^{m}$ whose values are imperfectly known and decrease in magnitude but slowly with increasing $m, n$. Now for many purposes it is of greater physical interest to know the resulting uncertainty in the locations and shapes of the magnetic contours than the uncertainties in the effective coefficients themselves. Here it is important to recognize that, although the value of a field component is a linear functional of the Gauss coefficients, and therefore of their uncertainties, the latitude-longitude locations at which a field component takes on a prescribed value is a highly nonlinear functional of the Gauss coefficients and their uncertainties. For these reasons it is both nontrivial and important to learn how inaccuracy in the Gauss coefficients and how the speed of convergence of truncated series affects the accuracy with which extrapolated magnetic contours can be located. Insofar as we are aware, this sensitivity question does not appear to have been approached previously in a systematic fashion (but see BoOKer, 1969). One limited aspect is treated below by examining an especially important contour map at different truncation levels.

Although several authors have presented contour maps of the extrapolated non-dipole field or of the equivalent scalar electric current function, there appear to be only two previously published extrapolated contour maps of the total field quantities presented here [Fig. 1 of Vestine et al. (1967), for epoch 1960, and Fig. 2 of Booker (1969) for epoch 1965, are contour maps of the radial field, at the core surface as in Fig. 2c below].

In the next section we present the maps and then intercompare then in Section 3. Concluding remarks are made in Section 4.

\section{The Contour Maps}

The maps analyzed here are grouped into three categories. In Fig. 1 (a, b, $\mathrm{c}, \mathrm{d})$ we plot contours of $X\left(=-B_{\theta}\right), Y\left(=B_{\phi}\right), Z\left(=-B_{r}\right), F(=|B|)$ at the surface of the earth, $r=a$, for epoch 1965.0 with $N=8$, using the IGRF (ZMUDA, 1971). Negative contours are plotted as dashed lines for clarity. The contour levels, expressed in units of $10^{-4} \mathrm{~T}(=1$ gauss), are basically the same as those seen in the IGRF figures. However, these plots have been prepared at the computing facility of the National Center for Atmospheric Research using a square array $(181 \times 181)$ of equally spaced latitude-longitude points, rather than the map projection in the World Magnetic Survey. The grid spacing is $1^{\circ}$ in latitude, $2^{\circ}$ in longitude. On each map a + and an $\times$ designate where, respectively, the maximum and minimum of that function occurs, the numerical values and coordinates being given in each caption. 
The maps in Fig. 1 not only constitute a check on the computations, but are also needed for purposes of comparison. The choice of the IGRF model for these maps is not based upon considerations of its accuracy compared to other available models, but entirely because of its international acceptance and the archival characteristic of these maps.

In Fig. 2 the same maps reappear but now evaluated at the core surface where $r=b$ so that $a / b=1.8282$. Contour levels are again indicated in units of $10^{-4} \mathrm{~T}$. Figure 3 consists of maps of the "magnetic equators," i.e., contours on which $Z=0$ at the core surface according to the 1975.0 model of BARRACLOUGH et al. (1975) at the six truncation levels $N=7,8,9,10,11,12$. These particular

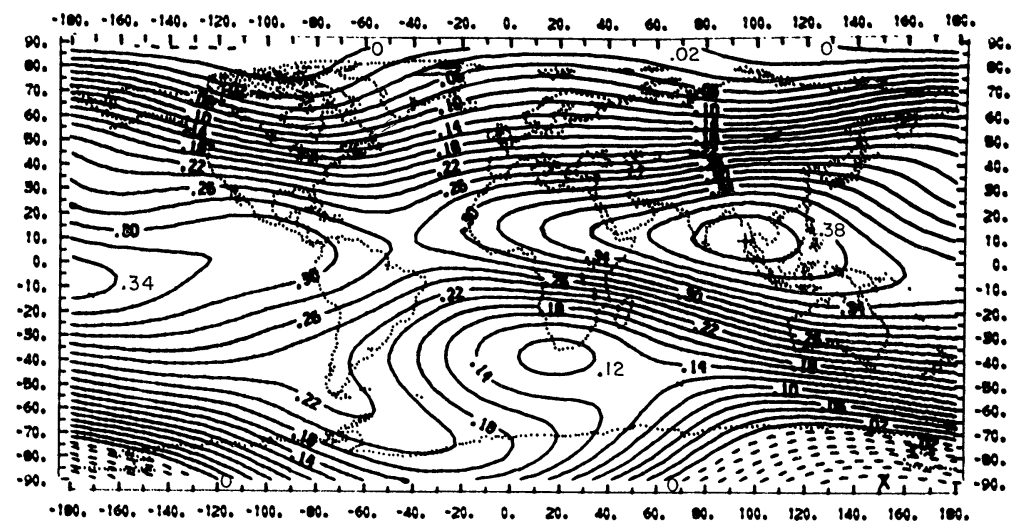

Fig. 1a. Geomagnetic contour map of $X$ component (north) at earth's surface for 1965 (IGRF), $N=8$, in units of $10^{-4} \mathrm{~T}\left(=1\right.$ gauss). $X_{\max }=4 \cdot 14 \cdot 10^{-5} \mathrm{~T}$ at $\theta=79^{\circ}, \phi=96^{\circ}$; $X_{\min }=-1.55 \cdot 10^{-5} \mathrm{~T}$ at $\theta=179^{\circ}, \phi=152^{\circ}$.

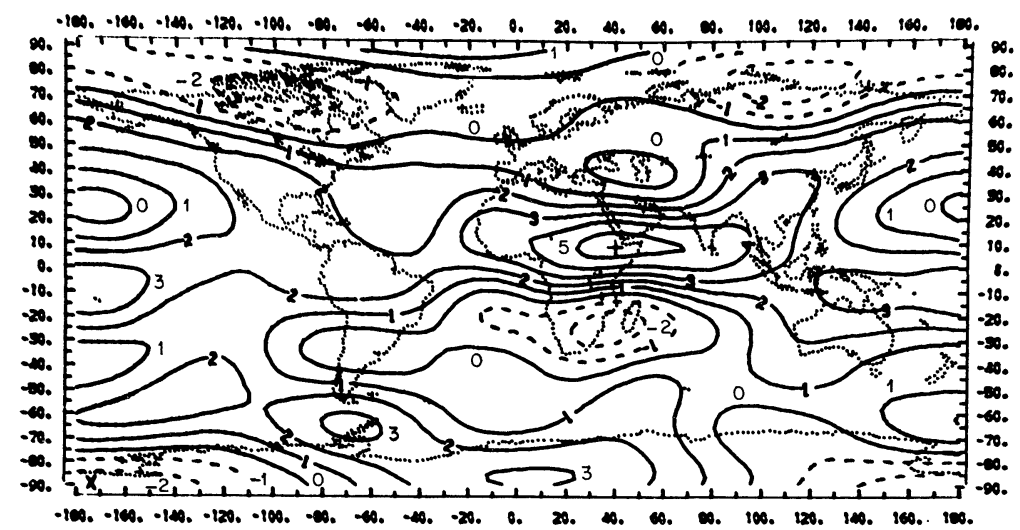

Fig. 2a. Geomagnetic contour map of $X$ component (north) at surface of the core for 1965 (IGRF), $N=8$, in units of $10^{-4} \mathrm{~T}\left(=1\right.$ gauss). $X_{\max }=5.45 \cdot 10^{-4} \mathrm{~T}$ at $\theta=80^{\circ}, \phi=$ $40^{\circ} ; X_{\min }=-2.87 \cdot 10^{-4} \mathrm{~T}$ at $\theta=179^{\circ}, \phi=-174^{\circ}$. 


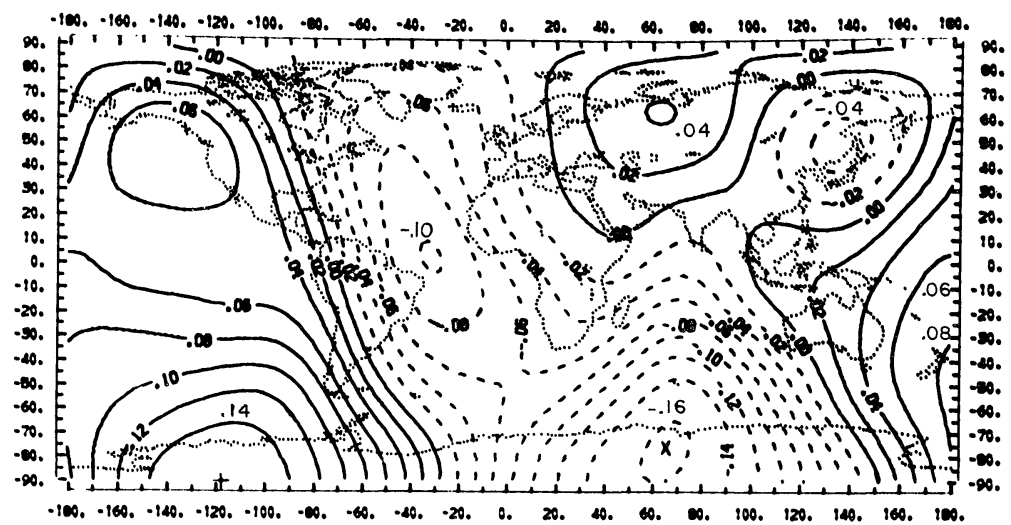

Fig. 1b. Geomagnetic contour map of $Y$ component (east) at earth's surface for 1965 (IGRF), $N=8$, in units of $10^{-4} \mathrm{~T}\left(=1\right.$ gauss). $\quad Y_{\max }=1.59 \cdot 10^{-5} \mathrm{~T}$ at $\theta=179^{\circ}, \phi=$ $-118^{\circ} ; Y_{\min }=-1.63 \cdot 10^{-5} \mathrm{~T}$ at $\theta=156^{\circ}, \phi=66^{\circ}$.

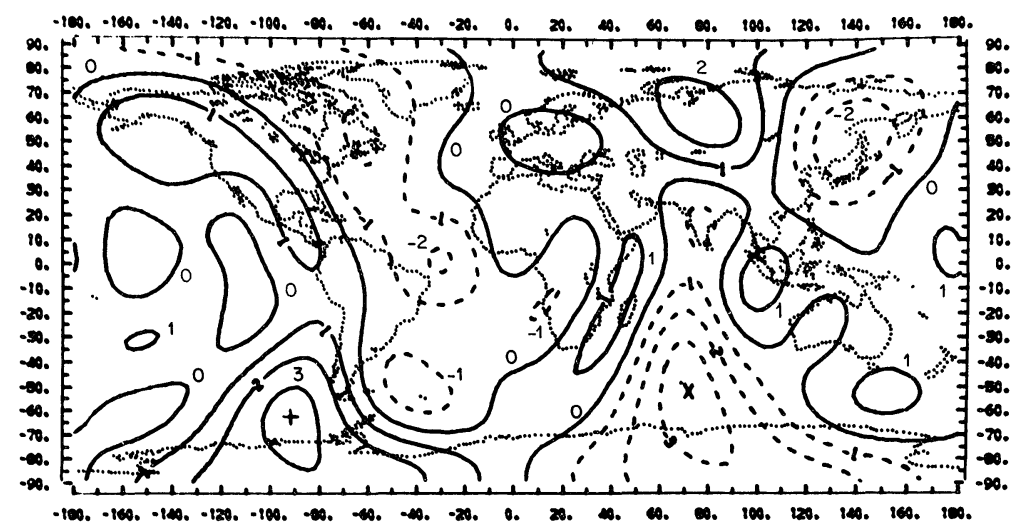

Fig. 2b. Geomagnetic contour map of $Y$ component (east) at surface of the core for 1965 (IGRF), $N=8$, in units of $10^{-4} \mathrm{~T}$ (=1 gauss). $Y_{\max }=-3.39 \cdot 10^{-4} \mathrm{~T}$ at $\theta=152^{\circ}$, $\phi=-92^{\circ} ; Y_{\min }=-3.57 \cdot 10^{-4} \mathrm{~T}$ at $\theta=142^{\circ}, \phi=72^{\circ}$.

contours possess a special physical significance in that they move horizontally with the fluid in a perfectly conducting core (BACKUS, 1968).

\section{Intercomparison of the Maps}

\subsection{Core surface and earth surface at common epoch (1965.0) and truncation level $(N=8)$}

It is of interest to compare contour maps at the surface of the core with those at earth's surface to see how the patterns change in scale upon extrapolation and how much the field components amplify in magnitude. 
At $r=a$, Fig. 1a shows that the contours of the northward field run predominantly east-west in the northern hemisphere but with greater meridional structure in the southern hemisphere. Fields with southward components are confined to extreme southern latitudes and are weak. This structure reflects the dominance of the axial dipole. Strongest northward fields occur in equatorial latitudes, the maximum value being $0.41 \cdot 10^{-4} \mathrm{~T}(=0.41$ gauss $)$ near Indochina. Some aspects of this pattern remain present at the core surface, Fig. 2a, but there are also quite noticeable differences. The maximum value of $5.45 \cdot 10^{-4} \mathrm{~T}$ representing an amplification by a factor of about 13 , is shifted $56^{\circ}$ to the west, beneath Africa. Regions of southward field now appear in the extreme latitudes

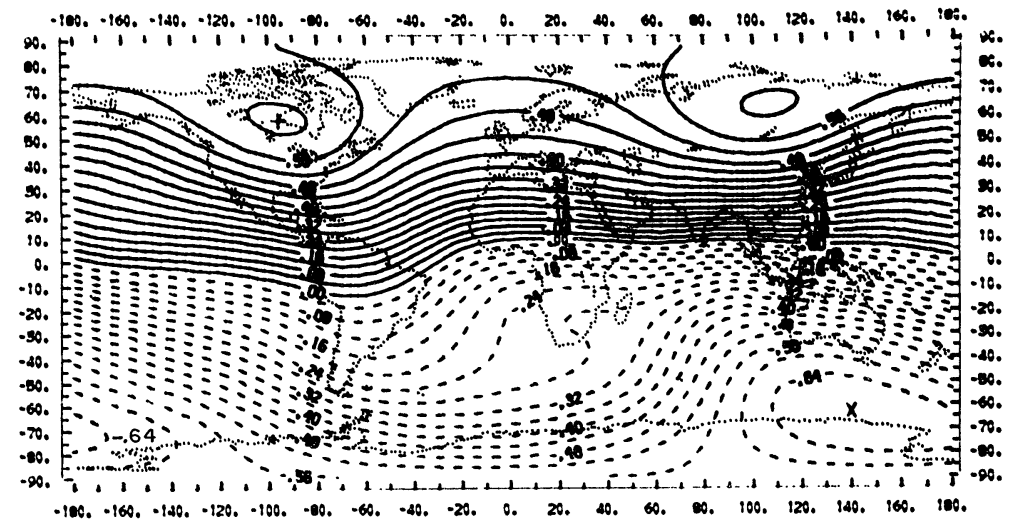

Fig. 1c. Geomagnetic contour map of $Z$ component (down) at earth's surface for 1965 (IGRF), $N=8$, in units of $10^{-4} \mathrm{~T}\left(=1\right.$ gauss). $Z_{\max }=6.06 \cdot 10^{-5} \mathrm{~T}$ at $\theta=31^{\circ}, \phi=-96^{\circ}$; $Z_{\min }=-6.78 \cdot 10^{-5} \mathrm{~T}$ at $\theta=153^{\circ}, \phi=140^{\circ}$.

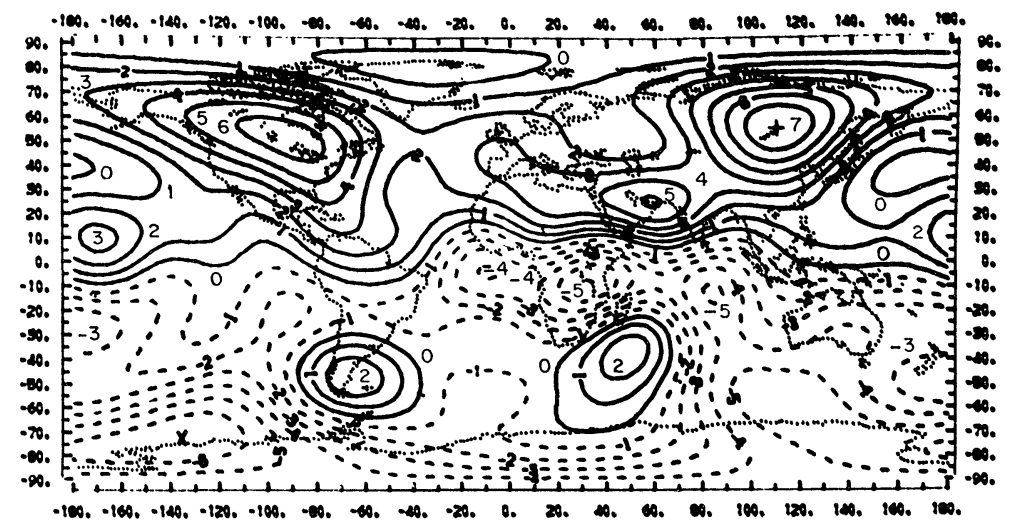

Fig. 2c. Geomagnetic contour map of $Z$ component (down) at surface of the core for 1965 (IGRF), $N=8$, in units of $10^{-4} \mathrm{~T}$ (=1 gauss). $Z_{\max }=7.90 \cdot 10^{-4} \mathrm{~T}$ at $\theta=34^{\circ}, \phi=$ $110^{\circ} ; Z_{\min }=-6.84 \cdot 10^{-4} \mathrm{~T}$ at $\theta=163^{\circ}, \phi=-136^{\circ}$. 


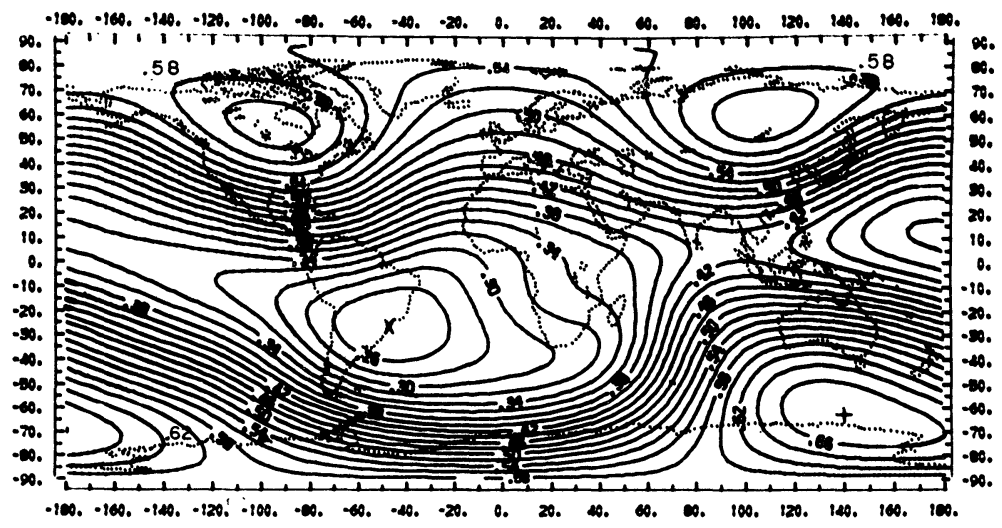

Fig. 1d. Geomagnetic contour map of $F$ (intensity) at earth's surface for 1965 (IGRF), $N=8$, in units of $10^{-4} \mathrm{~T}(=1$ gauss $) . F_{\max }=6.79 \cdot 10^{-5} \mathrm{~T}$ at $\theta=152^{\circ}, \phi=140^{\circ} ; F_{\min }=$ $2.40 \cdot 10^{-5} \mathrm{~T}$ at $\theta=116^{\circ}, \phi=-48^{\circ}$.

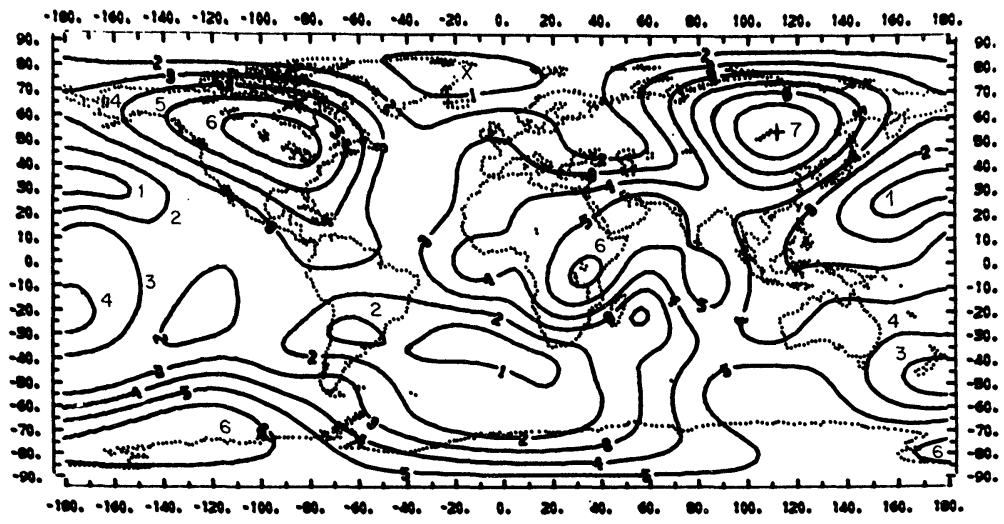

Fig. 2d. Geomagnetic contour map of $F$ (intensity) at surface of the core for 1965 (IGRF), $N=8$, in units of $10^{-4} \mathrm{~T}\left(=1\right.$ gauss). $F_{\max }=7.93 \cdot 10^{-4} \mathrm{~T}$ at $\theta=35^{\circ}, \phi=112^{\circ}$; $F_{\min }=2.30 \cdot 10^{-5} \mathrm{~T}$ at $\theta=13^{\circ}, \phi=-16^{\circ}$.

of both hemispheres as well as in a low latitude region extending from the eastern Atlantic into the Indian Ocean. The dipole is clearly no longer dominant, the typical structure superimposed on it being of continental scale (say, wave number 2 in latitude, 1 in longitude) compared with the still larger scale variations evident at earth's surface in Fig. 1a.

Figures $1 \mathrm{~b}$ and $2 \mathrm{~b}$ show that contours of the longitudinal field component tend to have rather more north-south orientation than the other components, and that such fields are comparatively weak. For an axial dipole such a field vanishes, and what shows up here is due both to the tilt of the best-fitting centered dipole relative to earth's rotation axis, as well as to non-dipole fields. 
Although there is definitely some smaller scale structure evident at the core surface, the contour pattern retains much of the gross structure seen at earth's surface. The agonic contour, $B_{\phi}=0$, through North and South America in Fig. $2 \mathrm{~b}$ is quite similar to that in Fig. 1b, but the Eastern hemisphere agonic contour is fragmented at the core surface. Regions of maximum and minimum longitudinal field component continue to occur in southern latitudes near the same longitudes as at $r=a$, with the height and depth of the maximum and minimum each being amplified by about the same factor of 21 .

The magnetic $Z$ component contour map, Fig. 2c, is especially significant since this reveals the regions where magnetic flux passes out of the core into the mantle. Furthermore, as first emphasized by BACKus (1968), the zero level

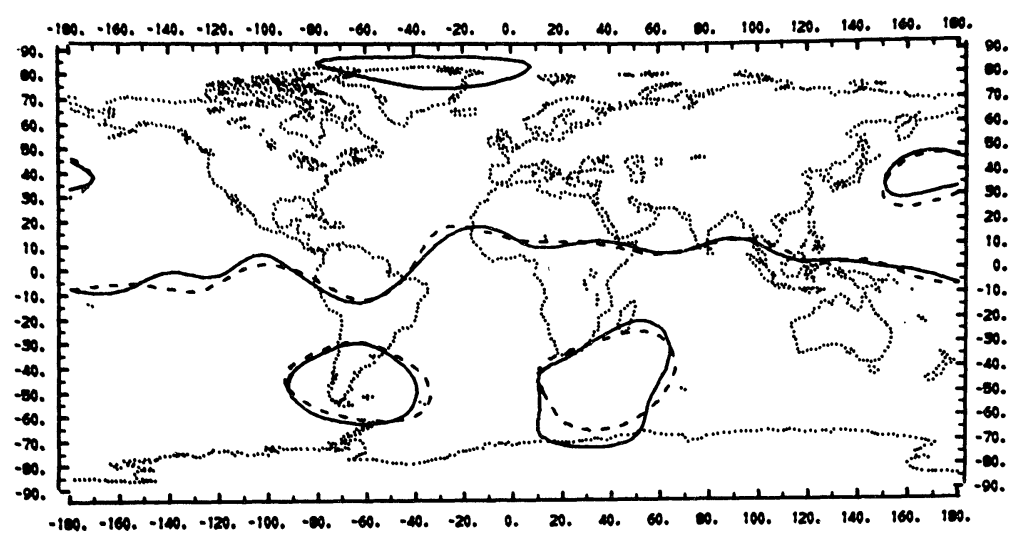

Fig. 3a. Null-flux contours $(Z=0)$ on the surface of the core for 1975 (BARRACLOUGH et al.). $N=7$ (dashed); $N=8$ (solid).

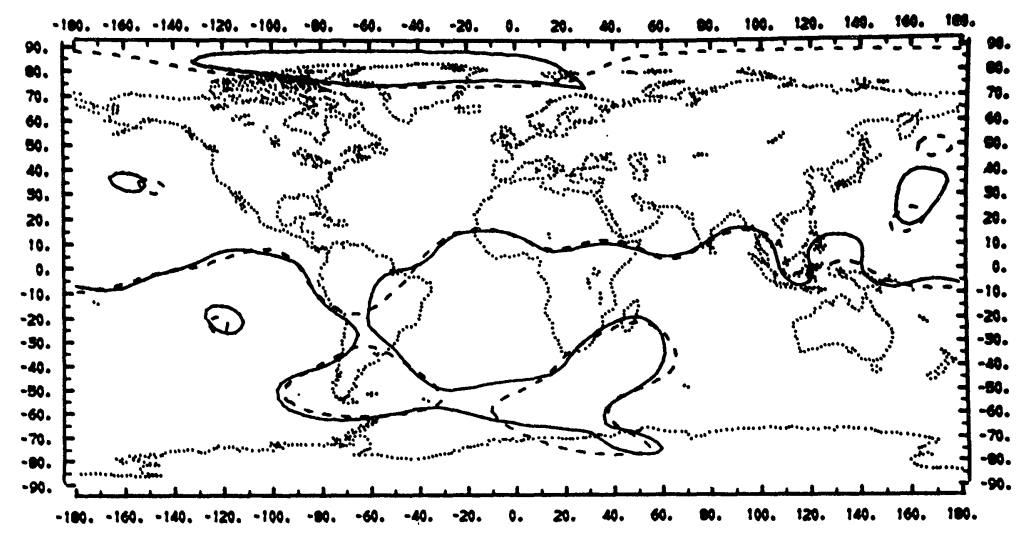

Fig. 3b. Null-flux contours $(Z=0)$ on the surface of the core for 1975 (BARRACLOUGH et al.). $N=9$ (dashed); $N=10$ (solid). 


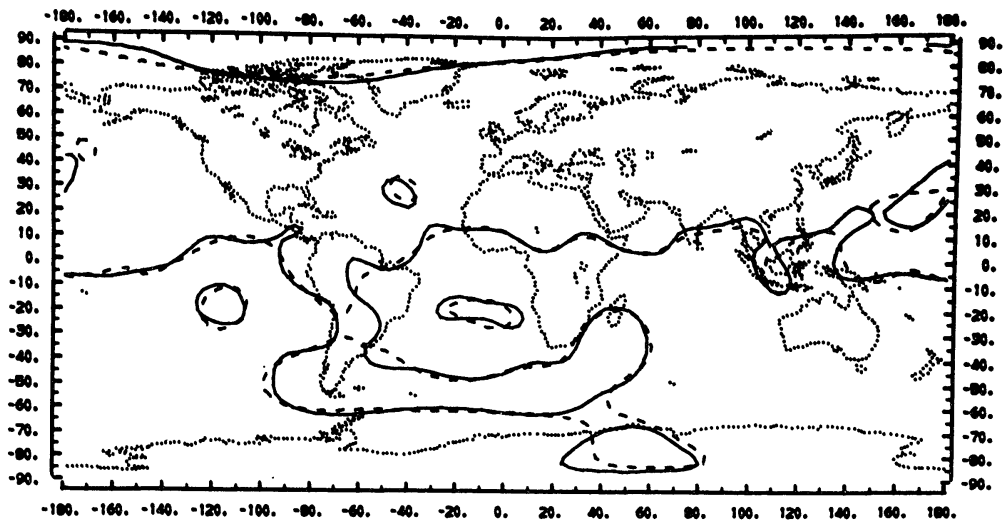

Fig. 3c. Null-flux contours $(Z=0)$ on the surface of the core for 1975 (BARRACLOUGH et al.). $N=11$ (dashed); $N=12$ (solid).

contours of the $Z$ field (see Fig. 3) move with the core fluid on time scales short enough that resistive diffusion is ignorable.

At earth's surface, Fig. 1c, most of the $Z$ contours encircle the poles with strong east-west trends, apart from two symmetric regions of local maximum (of about equal strength and latitude) in the northern hemisphere, a slightly stronger minimum off of Antarctica, and a weak "disturbance" in southern Africa. These three regions of local maximum or minimum vertical field can still be identified at the core-mantle boundary, Fig. $2 c$, but in addition there is a pronounced local minimum beneath Africa and two local highs in the southern hemisphere not evident at earth's surface. Although there is only one zero level contour at $r=a$ across which the vertical field reverses sense (the magnetic equator), there are five "null-flux curves" (BACKUS, 1968) on the surface of the core (for a view of these "magnetic equators" alone refer to Fig. 3a). The globe-encircling main magnetic equator, the South American, the South African, and the Pacific null-flux curve were each present in the study made by Booker (1969). However, here there is also a null-flux curve in the Arctic, not seen on Booker's maps. This is presumably due to his truncation of a Mercator projection at about the latitude where that contour begins. However, since the area enclosed by that contour is only about $1 / 250$ th of the area of the core-mantle boundary, the shape, location, and even real existence of this contour cannot be regarded as well-determined by a field model with $N=8$ (for which there are only 80 Gauss coefficients, BACKUs, 1968; BooKer, 1969). We return to this difficulty shortly.

The overall pattern of $Z$ contours at earth's surface, Fig. 1c, with downward (upward) flux in the northern (southern) hemisphere divided by the gently undulating main magnetic equator is due to the dominance of an off-center, 
tilted dipole as the leading order field at earth's surface. The first departure from this simple behavior, giving extra regions of local maximum or minimum, reflect the importance of the quadrupole and octupole terms in (1). At the core surface, Fig. 2c, the main magnetic equator has much the same shape as at earth's surface, but with some superimposed wave-like structure of typical length scale $1 / 10$ of the circumference of the equator on the core surface (i.e., about $2 \cdot 10^{6} \mathrm{~m}$ ). The maximum downward field (of $7.9 \cdot 10^{-4} \mathrm{~T}$ ) occurs in Siberia at $r=b$, rather than in North America, as at $r=a$ and the amplification factor is about 13 .

Contour maps of total field strength, $F$, are given in Figs. 1d, 2d. At earth's surface, in non-equatorial latitudes, the contours are much like those of the vertical field, Fig. 1c (with appropriate sign change in the southern hemisphere) because of the comparative weakness of both horizontal field components (Figs. 1a, 1b). The local minimum field intensity of $0.24 \cdot 10^{-4} \mathrm{~T}$ in South America arises mostly from an amalgamation of the $X$ and $Z$ fields (the $Y$ field is relatively weak in that region). The two northern hemisphere regions of local maximum field strength and the local minimum known as the South Atlantic anomaly, recur at the surface of the core; but there are, in addition, new regions of low field strength just east of Greenland and in the central Pacific. The absolute maximum of $7.93 \cdot 10^{-4} \mathrm{~T}$ in Siberia is an amplification by a factor of about 12 over the maximum field intensity at earth's surface, which occurs south of Australia.

\subsection{Effect of truncation level $N$ at core surface for common epoch (1975.0)}

Because useful descriptions of the field can be based on the contour maps in question, it becomes important to know just how well or how poorly the contours are determined by geomagnetic field models. As noted in Section 1, this is believed to be a relatively untouched problem. Here, we make a preliminary evaluation in terms of the single contour map showing null-flux curves at the core surface. The twelfth order, twelfth degree model for 1975.0 constructed by BARRAClough et al. (1975) has been utilized for this purpose. Figures $3 \mathrm{a}, 3 \mathrm{~b}, 3 \mathrm{c}$ give the maps for the six truncation levels $N=(7,8),(9,10)$, $(11,12)$, respectively. Since, in theory, these null-flux curves connect the same fluid particles for time durations of decades, their number and topology ought to be preserved in time. Comparison of the zero level contours in Fig. 2c with the solid curves in Fig. 3a shows that this condition was met for $N=8$ between 1965 and 1975. However, at a single epoch, there is a substantial dependence on $N$ of the numbers and topology of null-flux curves. For example, while null-flux curves at $N=7,8$ in Fig. 3 a are quite similar (apart from the absence of the poorly resolved Arctic null-flux curve at $N=7$ ), beyond $N=8$ 
small scale erratic structures become evident. The main magnetic equator, the South American and South African loops appear to be fairly well represented in all models up through $N=9$. The Pacific loop at $N=8$ encloses an area of about $1 / 90$ th of the surface area of the core so it is probably a bit beyond the resolving power of the model. It is not then surprising to find its position and shape sensitive to $N$. In contrast, the South American and African loops contain about $1 / 45$ th and $1 / 30$ th, respectively, of the core surface area at $N=8$ so they could be reasonably stable to changes in $N$; yet they are not. The fact that an Arctic null-flux curve appears for all of the values of $N$ plotted except $N=7$ is, perhaps, an indication that it truly exists, but its location is not well determined. Even the location of the main magnetic equator is not especially independent of $N$ for $N>9$. Although these results are not surprising on theoretical grounds, they do emphasize the desirability of developing nonlinear data fitting techniques in which, instead of best-fitting to give field values at all points of the globe, one rather chooses the Gauss coefficients so as to maximize the accuracy with which individual contours are located, and, is possible, to minimize the sensitivity to truncation level. This will be the subject of future work.

One quantitative test of the speed with which the truncated series representation is converging is provided by the results compiled in Table 1 . The latitude and longitude at which the radial field takes on its absolute maximum value at the core surface, as well as the maximum value are tabulated for $N=$ $7, \ldots, 12$ (using the 1975 model of Barraclough et al.). The latitude of maximum $B_{r}$ is stable to within $\pm 4^{\circ}$ and the longitude of maximum $B_{r}$ appears to be close to $110^{\circ}$. Entirely similar results are found in terms of the place where the total field intensity reaches its maximum value. It is worth noting that at $N=12$ this model predicts maximum field strengths (and maximum radial fields) very near $10^{-3} \mathrm{~T}$ at the core surface. This is at least twice as great as the value usually quoted in the literature (e.g., RoBerTs and SowARD, 1972), and so the local poloidal magnetic energy may reach values four times as large as previously thought.

It is also clear from Table 1 that the position of maximum $B_{r}$ is considerably better determined than the positions where $B_{r}=0$. This enhanced sensitivity

Table 1. The effect of truncation level $N$ on the value of $B_{r}$ and location where $B_{r}$ reaches its maximum on the core surface according to the 1975 model of Barraclough et al.

\begin{tabular}{lrrrrrr}
\hline \multicolumn{1}{c}{$N$} & 7 & 8 & 9 & 10 & 11 & 12 \\
\hline$\beta_{\max }=90-\theta_{\max }$ (deg.) & 59 & 57 & 65 & 65 & 62 & 60 \\
$\phi_{\max }($ deg.) & 112 & 110 & 114 & 110 & 110 & 110 \\
$B_{r_{\max }\left(10^{-4} \mathrm{~T}\right)}$ & 7.01 & 7.83 & 8.18 & 8.75 & 9.19 & 9.92 \\
\hline
\end{tabular}


to $N$ of the location of null-flux curves is reminiscent of the "perpendicular error effect" of Lowes (1975). Such model dependence will have to be reduced if reliable results from geomagnetic contour maps are to be obtained.

The authors are indebted to the Computing Facility of the National Center for Atmospheric Research which is sponsored by the National Science Foundation for providing the computer time needed to produce the contour maps. This work has been supported by the Division of Earth Sciences of the National Science Foundation under NSF Grant DES 75-03960.

\section{REFERENCES}

BaCKUS, G.E., Kinematics of geomagnetic secular variation in a perfectly conducting core, Phil. Trans. Roy. Soc. Lond. A, 263, 239-266, 1968.

Barraclough, D.R., J.M. Harwood, B.R. Leaton, and S.R.C. Malin, A model of the geomagnetic field at epoch 1975, Geophys. J. R. Astr. Soc., 43, 645-659, 1975.

Benton, E.R., On fluid circulation around null-flux curves at earth's core-mantle boundary, Geophys. Astrophys. Fluid Dyn., 11, 323-327, 1979.

Booker, J.R., Geomagnetic data and core motions, Proc. Roy. Soc. Lond. A, 309, 27-40, 1969.

Busse, F.H., A model of the geodynamo, Geophys. J. R. Astr. Soc., 42, 437-459, 1975.

Busse, F.H., Magnetohydrodynamics of the earth's dynamo, Ann. Rev. Fluid Mech., 10, 435462, 1978.

Engdahl, E.R. and L.E. Johnson, Differential PcP travel times and the radius of the core, Geophys. J. R. Astr. Soc., 39, 435-456, 1974.

Lowes, F.J., Spatial power spectrum of the main geomagnetic field and extrapolation to the core, Geophys. J. R. Astr. Soc., 36, 717-730, 1974.

Lowes, F.J., Vector errors in spherical harmonic analysis of scalar data, Geophys. J. R. Astr. Soc., 42, 637-651, 1975.

Moffatt, H.K., Generation of magnetic fields by fluid motions, Adv. Appl. Mech., 16, 119$181,1976$.

RoberTs, P.H. and S. ScotT, Truncation errors in the spherical harmonic analysis of the geomagnetic field and the problem of downward extrapolation, J. Geomag. Geoelectr., 15, $148-160,1964$.

Roberts, P.H. and S. Scotr, On analysis of the secular variation, 1. A hydromagnetic Constraint: Theory, J. Geomag. Geoelectr., 17, 137-151, 1965.

Roberts, P.H. and A.M. SowArd, Magnetohydrodynamics of the earth's core, Ann. Rev. Fluid Mech., 4, 117-154, 1972.

SowARD, A.M. and P.H. Roberts, Recent developments in dynamo theory, Magnitnaya Gidrodinamika, 1, 3-51, 1976.

Vestine, E.H., R.H. BALL, and A.B. KAHLE, Nature of surface flow in the earth's central core, J. Geophys. Res., 72, 4927-4936, 1967.

ZMUdA, A.J. (editor), World Magnetic Survey 1957-1969, IAGA Bulletin No. 28, IUGG Publication Office, Paris, 1971. 